\title{
Low-Profile Dual Circularly Polarized Antenna Array for Satellite Communications in the X Band
}

\author{
Andrés García-Aguilar, José-Manuel Inclán-Alonso, Lucía Vigil-Herrero, José-Manuel Fernández-González, and \\ Manuel Sierra-Pérez, Senior Member, IEEE
}

\begin{abstract}
A planar antenna is introduced that works as a portable system for $\mathrm{X}$-band satellite communications. This antenna is low-profile and modular with dimensions of $40 \times 40 \times 2.5 \mathrm{~cm}$. It is composed of a square array of 144 printed circuit elements that cover a wide bandwidth $(14.7 \%)$ for transmission and reception along with dual and interchangeable circular polarization. A radiation efficiency above $50 \%$ is achieved by a low-loss stripline feeding network. This printed antenna has a $3 \mathrm{~dB}$ beamwidth of $5^{\circ}$, a maximum gain of $26 \mathrm{dBi}$ and an axial ratio under $1.9 \mathrm{~dB}$ over the entire frequency band. The complete design of the antenna is shown, and the measurements are compared with simulations to reveal very good agreement.
\end{abstract}

Index Terms-Antenna arrays, dual-polarized antennas, microstrip antennas, satellite communication.

\section{INTRODUCTION}

$\mathbf{N}$ EW fixed and mobile satellite systems [1] require antenna systems that must be portable, low-profile and lightweight to provide ubiquitous and high-capacity communications-on-the-move. Planar antennas are perfect candidates for meeting these specifications. Slots [2] are often used as radiating elements, but microstrip patches [3] are preferable due to their ease of manufacturing and lower weight. Although phased arrays [4], [5] can reconfigure an antenna system to point in a desired direction, low-cost systems are in high demand. Hybrid mechanical/electronic steerable solutions reduce costs [6] but are more expensive and difficult to design than a fixed beam array antenna. The main goal of this research is to design and measure a printed array antenna that could be used as a portable man-pack with a high efficiency and cover a wide frequency band for personal satellite communication services.

Microstrip arrays exhibit low efficiencies (typically less than $50 \%$ ) due to ohmic/dielectric losses and parasitic radiation in

Manuscript received June 27, 2011; revised September 16, 2011; accepted October 26, 2011. Date of publication March 09, 2012; date of current version May 01, 2012. The simulations performed in this work were realized using CST Microwave Studio Suite 2011 under a cooperation agreement between Computer Simulation Technology (CST) and the Technical University of Madrid (UPM). The project was led by the Technical University of Madrid and Antenas Moyano S.L. with the support of the Spanish Education Ministry (Comisión Interministerial de Ciencia y Tecnología) under reference TEC2008-06736/TEC and a Spanish Government Grant (FPI under reference BES-2009-021462). This work was supported in part by the U.S. Department of Commerce under Grant BS123456.

The authors are with the Radiation Group, Technical University of Madrid (Universidad Politécnica de Madrid-UPM), Madrid 28040, Spain (e-mail: andresg@gr.ssr.upm.es; chema@gr.ssr.upm.es; lucia@gr.ssr.upm.es; jmfdez@gr.ssr.upm.es; m.sierra.perez@gr.ssr.upm.es).

Color versions of one or more of the figures in this paper are available online at http://ieeexplore.ieee.org.

Digital Object Identifier 10.1109/TAP.2012.2189729 the feeding network and also due to surface wave excitations in the dielectric substrate [7], [8]. Some serial waveguide-fed structures [9] are able to obtain higher efficiencies but increase the weight and provide narrow bandwidths. In this work, by designing a low-loss stripline corporative network and performing active impedance matching for the microstrip patch, the measured radiation efficiency remains between $50 \%$ and $60 \%$, depending on the frequency and polarization. This antenna works in the X band from 7.25 to $8.4 \mathrm{GHz}$, which represents a $14.7 \%$ relative bandwidth.

The antenna was designed to be planar, compact, modular and dual circular polarized for transmission (Tx) and reception $(\mathrm{Rx})$ bands simultaneously. It is composed of a square planar array of $12 \times 12$ double-stacked microstrip patches fed by two via holes and a hybrid circuit to create two orthogonal modes in the microstrip patch and the dual circular polarization [10]. Moreover, to reinforce the circular polarization the sequential rotation technique is used between $2 \times 2$ patches. The array is divided into 9 identical subarrays of $4 \times 4$ radiating elements, making it possible to separate the manufacturing of the subarrays from that of the global signal distribution network, simplifying the corporative network and providing a modular structure suitable to a serial fabrication process. Planar multilayer antenna technology [11], [12] is appropriate for new subarray antenna architectures that integrate the radiating elements with the associated active and passive circuitry. In this system, each subarray forms a multilayer board, making it simple to update the antenna with electronic scanning capabilities.

The subarray feeding network is fabricated using microstrip technology for right-handed and left-handed circular polarization (RHCP and LHCP, respectively). The global distribution network is a low-loss stripline located under the subarrays. This type of feeding network involves a trade-off between the simplicity of exciting the radiating elements using printed circuits and loss reduction by separating the distribution network into a structure designed to realize low losses. The power distribution network is connected to each subarray through SMP-type coaxial connectors, and there are two global ports (input/output) with SMA-type connectors, one for each polarization.

Table I summarizes the main characteristics of the antenna.

The paper is organized as follows. Section II describes the composition and the different layers of this antenna system. Section III presents the subarray design and measurements of the radiating element, the hybrid circuit and the array unit cell. In Section IV, the design and measurements of the stripline corporative network are shown. Section V shows measurements of the prototyped antenna. Finally, conclusions are drawn and presented in Section VI. 
TABLE I

ANTENNA SPECIFICATIONS

\begin{tabular}{ccc}
\hline \hline Parameter & Specification & Units \\
\hline $\begin{array}{c}\text { Working bands } \\
\text { Tx: } \\
\text { Rx: }\end{array}$ & $\begin{array}{c}7.9 \text { to } 8.4 \\
7.25 \text { to } 7.75\end{array}$ & $\begin{array}{c}\mathrm{GHz} \\
\mathrm{GHz}\end{array}$ \\
\hline Polarization & $\begin{array}{c}\text { Dual circular polarization } \\
\text { for Tx and Rx bands }\end{array}$ & - \\
\hline 3dB beamwidth & 5 & degrees \\
\hline Maximum gain & 26 & $\mathrm{dBi}$ \\
\hline Radiation efficiency & $50-60$ & $\%$ \\
\hline Axial Ratio & $<1.9$ & $\mathrm{~dB}$ \\
\hline $\begin{array}{c}\text { Isolation between Tx } \\
\text { and Rx }\end{array}$ & $>20$ & $\mathrm{~dB}$ \\
\hline $\begin{array}{c}\text { Maximum Side Lobe } \\
\text { Level }\end{array}$ & -13 & $\mathrm{~dB}$ \\
\hline Size & $40 \times 40 \times 2.5$ & $\mathrm{~cm}$ \\
\hline Weight & 3.6 & $\mathrm{~kg}$ \\
\hline
\end{tabular}

\section{ANTENNA DESCRIPTION}

The antenna is formed by an array of $12 \times 12$ microstrip patches and is divided into 9 equal subarrays of $4 \times 4$ elements. The distance between patches is $33 \mathrm{~mm}$. Fig. 1 shows a schematic of the array composition and the different layers of the antenna. The upper layer corresponds to the subarrays, while the bottom layer is the excitation network. The former has a microstrip technology feeding structure with one layer for RHCP and LHCP that excite the microstrip patches through two via holes. The patch and subarray network layers exhibit a unique structure in which they are interconnected to the global distribution network through two SMP-type coaxial connectors. To provide robustness, a $5 \mathrm{~mm}$ foam sheet was added above the upper dielectric layer. The global power distribution network is composed of a stripline layer for RHCP and LHCP and two general SMA input/output ports, one for each polarization. The stripline network is designed for low losses and is protected by walls on both sides to prevent the excitation of undesired modes. The antenna is designed for a reference input impedance of $50 \Omega$.

To achieve a lower side lobe level (SLL), excitation tapering was implemented in the subarrays. Thus, the signal amplitudes of each subarray are not equal but do have symmetry with respect to the two main antenna axes.

\section{SUBARRAY CONFIGURATION}

\section{A. Radiating Element, Hybrid Circuit and Unit Cell}

The radiating element in Fig. 2 is a double-stacked microstrip patch that covers a wide frequency band and is excited by two via holes with a branch-line coupler (BLC) to generate circular polarization [10]. This printed element must work over a large bandwidth (14.7\%), and therefore a thick lower substrate (1.58 $\mathrm{mm}$ ) is required. The dielectric supports surface waves that will degrade the antenna efficiency by increasing the coupling between the array elements. Nevertheless, such a coupling can be compensated by designing the radiating element in an array environment and supposing that the element is immersed in an infinite two-dimensional periodic structure. This assumption is valid for the central radiating elements in the real array. It will

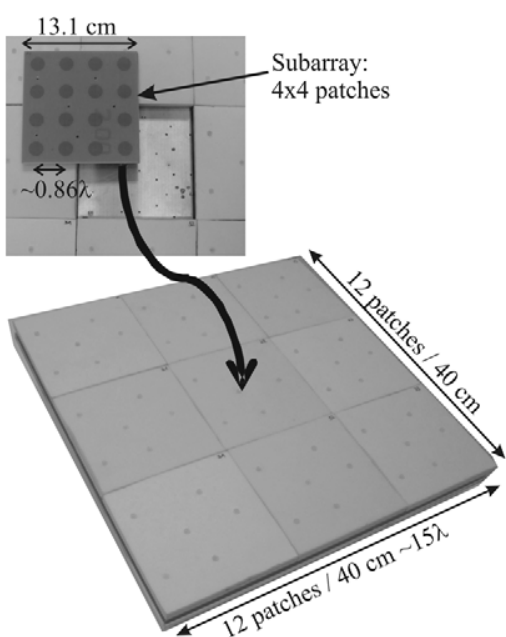

(a)

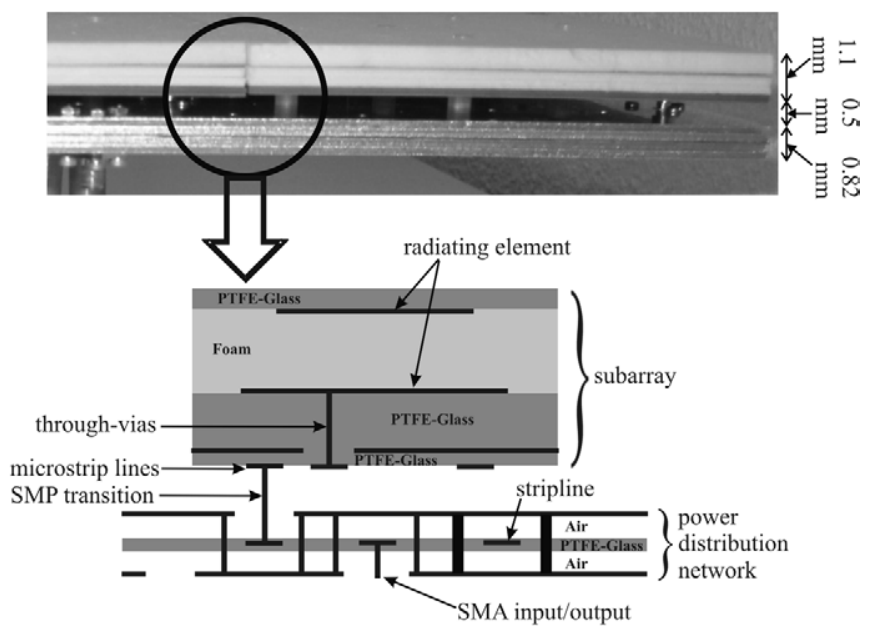

(b)

Fig. 1. Printed antenna structure. (a) Perspective view with subarray detail. (b) Side view and multilayer scheme.

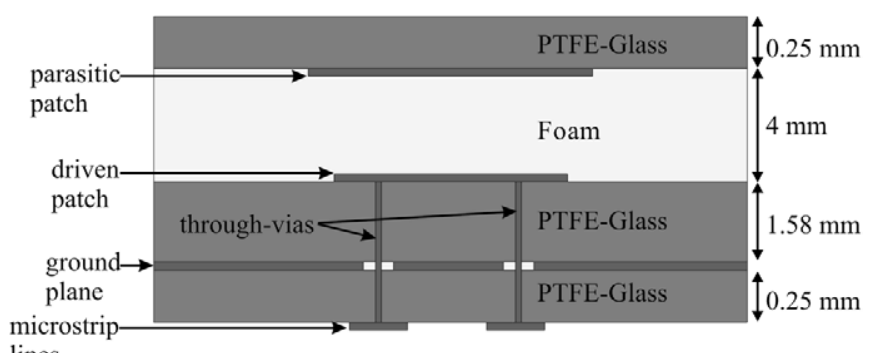

(a)

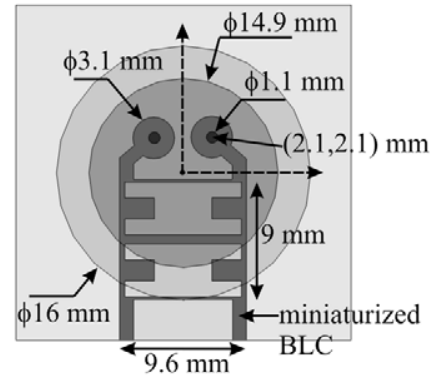

(b)

Fig. 2. Radiating element. (a) Side view. (b) Bottom view. 


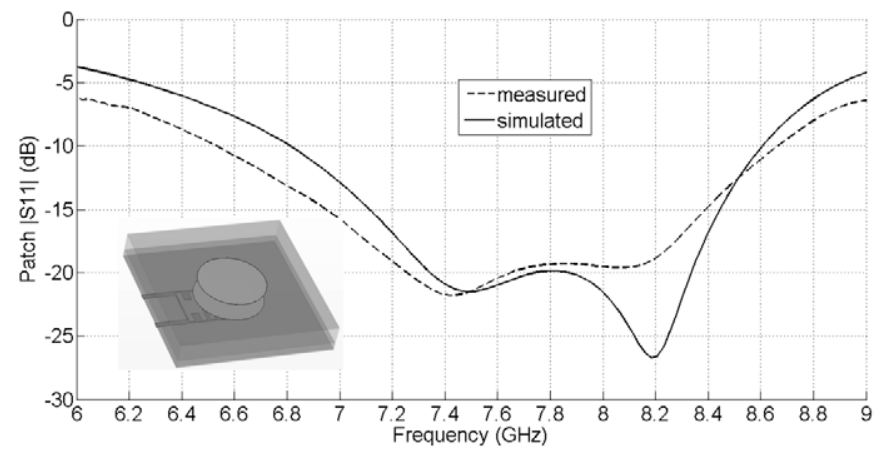

Fig. 3. Isolated radiating element input reflection coefficient.

be less accurate for the edge patches but is sufficient for the design of this antenna, as will be shown later. Other options are available to reduce the couplings, such as using an impedance matching network in the microstrip feeding structure [13], designing a cavity microstrip antenna as a radiating element [14] or including a structure with a forbidden frequency band [15] to cut off dielectric surface waves.

The double-stacked patch optimization is done using a FDTD full-wave simulator where the thickness of the substrates is kept fixed and the patch diameters (that control the frequency resonance) and the via hole positions (that match the microstrip feeding network and the patch impedances) are the optimization parameters. The goal is to achieve an input reflection coefficient lower than $-15 \mathrm{~dB}$, and an axial ratio better than $3 \mathrm{~dB}$. Periodic boundary conditions are used to optimize the element within an infinite planar array.

The radiating element is designed for a reference impedance of $40 \Omega$ due to a restriction in the fabrication of the minimum line width, because one of the BLC microstrip lines is in the manufacturing limit of $0.2 \mathrm{~mm}$. Fig. 3 exhibits the simulated and measured reflection coefficient for an isolated radiating element, where a very good agreement is met. Thus, it is shown that one single element can cover the working bandwidth properly.

The BLC can work with dual circular polarization, i.e., with simultaneous RHCP and LHCP, in the same band as the patches. Thus, a three-branch periodic $3 \mathrm{~dB}$ BLC was designed [16] to cover the same bandwidth. However, a conventional configuration requires an area of $13.2 \mathrm{~cm}^{2}$, which is large compared to the radiating element and the subarray; therefore, this BLC was miniaturized using the equivalence between a $\lambda / 4$ transmission line and a line with an open-ended shunt stub [17]. Other configurations have been investigated, including fractal shapes [18] and interdigitally-coupled lines [19], but this solution was chosen due to its simplicity and quality. The compact BLC is shown in Fig. 2(b), and Fig. 4 presents a comparison between the simulated and measured S-parameters. The BLC works properly, and the discrepancies between simulations and measurements are caused by the SMP connectors, whose effect is not included in simulations. The area is reduced by about $35 \%$, diminishing the couplings inside the subarray feeding network.

To improve the circular polarization quality, the sequential rotation technique [10] was implemented in each $2 \times 2$ element, shown in Fig. 5. Each patch was rotated $90^{\circ}$ and excited by a

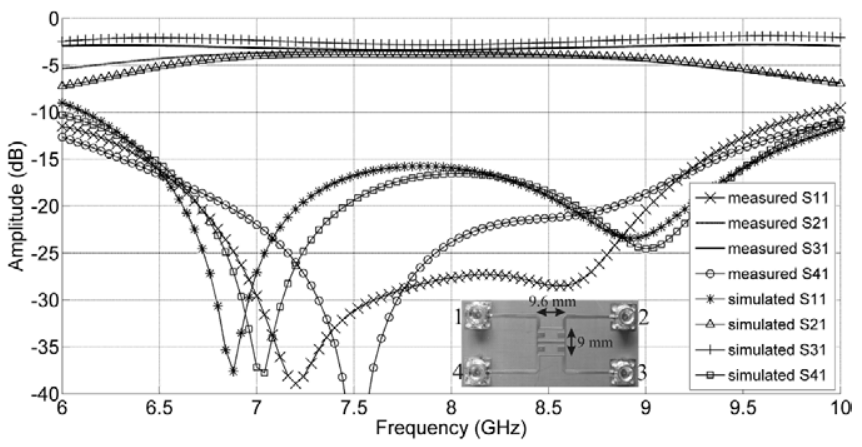

(a)

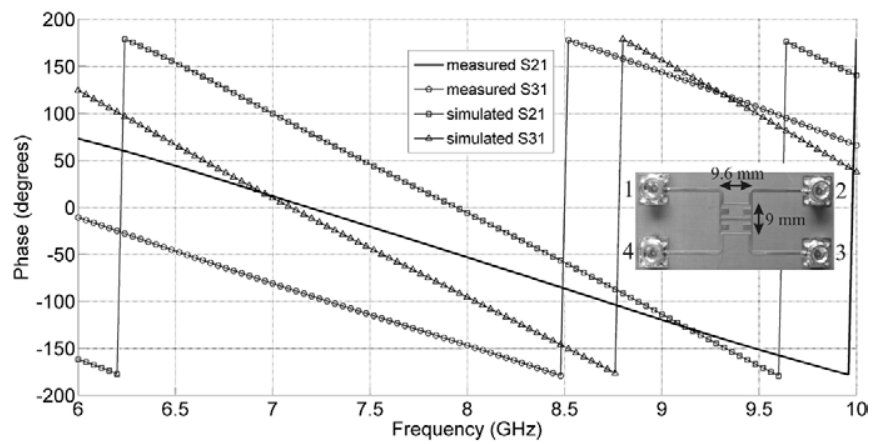

(b)

Fig. 4. Miniaturized BLC S-parameter measurements. (a) Amplitude (b) Phase.
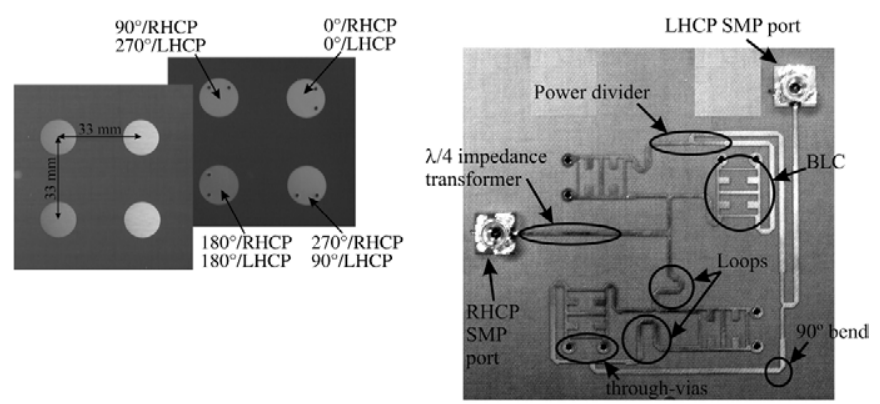

(a)

(b)

Fig. 5. Unit cell. (a) Stacked microstrip patches. (b) Microstrip feeding network.

signal phase-shifted $90^{\circ}$ relative to that of the previous element, as indicated in Fig. 5(a). These $2 \times 2$ radiating elements form the array unit cell. Such a configuration allows an improvement of approximately $20 \mathrm{~dB}$ in the copolar-crosspolar ratio.

\section{B. Subarray}

For simplicity, subarrays have $4 \times 4$ radiating elements, and because the antenna size is a predefined requirement, two interelement spacing are possible: a distance of $25 \mathrm{~mm}(\sim 0.65 \lambda)$, where the array has $16 \times 16$ elements with $4 \times 4$ subarrays; and a separation of $33 \mathrm{~mm}(\sim 0.86 \lambda)$, with an array of $12 \times 12$ elements and $3 \times 3$ subarrays. The latter is chosen because it allows an easier design of the subarray and their feeding lines, and requires less connections between the subarrays and the global feeding network. On the other hand, the former presents lower SLL, but this difference is not very severe and is only important for angles far away from the normal direction of the antenna. 


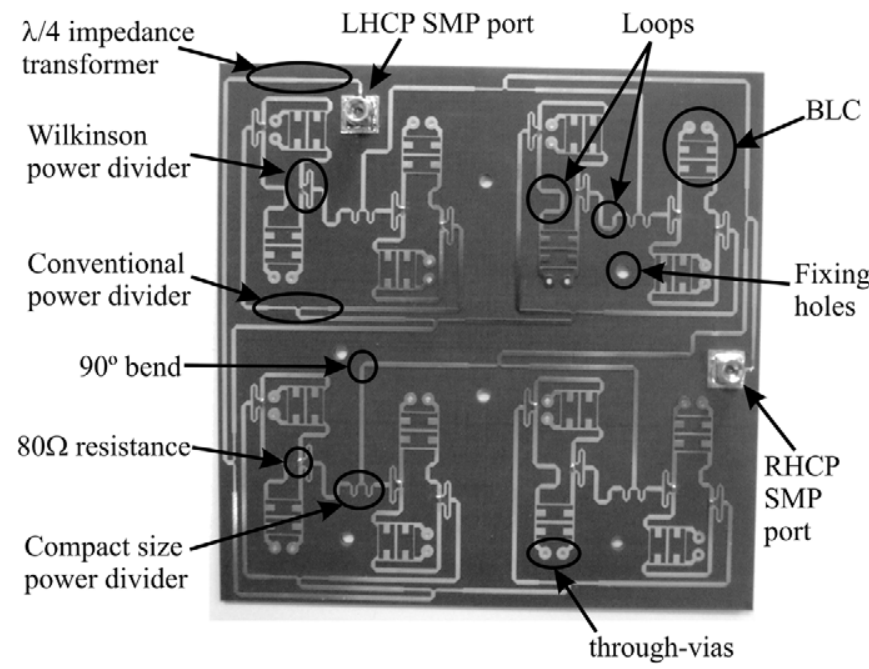

Fig. 6. Subarray microstrip feeding network.

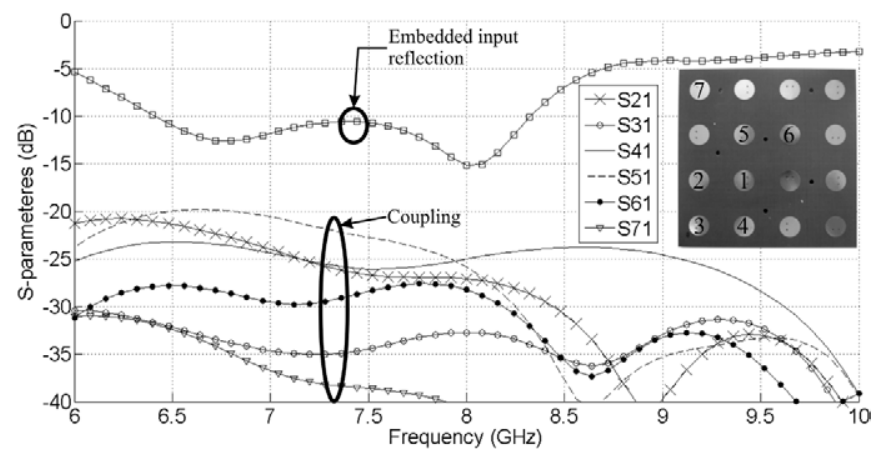

Fig. 7. Simulated embedded input reflection and coupling coefficients among some subarray elements for RHCP.

Hence, there are 9 equivalent subarrays with a total size of $13.1 \times 13.1 \times 0.61 \mathrm{~cm}$ that comprise a unique multilayer board and are interconnected with a power distribution network through SMP connectors (surface mount) and vertical transitions. Such connectors and vertical transitions exhibit measured transmission losses of approximately 1 to $2 \mathrm{~dB}$, which will reduce the realized gain in the radiation pattern measurements compared with simulations, in which their effects have not been considered.

Fig. 6 shows the subarray feeding network prototype. Unit cell is repeated four times and joined together in phase through two different paths, one for each polarization. Loops help to carry the correct phase at each point regarding with the sequential rotation and the polarization. Wilkinson power dividers before the BLC eliminate reflected signals from the patches, and compact size conventional power dividers reduce the couplings among the microstrip lines. $\lambda / 4$ impedance transformers are placed at each SMP port to change the line characteristic impedance from $40 \Omega$ to the final $50 \Omega$.

Fig. 7 depicts the simulated input match for a central element within the subarray, as well as the coupling among surrounding patches, where microstrip network has not been included. Coupling coefficients between radiating elements are kept under $-20 \mathrm{~dB}$ revealing that this effect is not increasing the losses in the antenna due to surface waves propagation. Mainly,

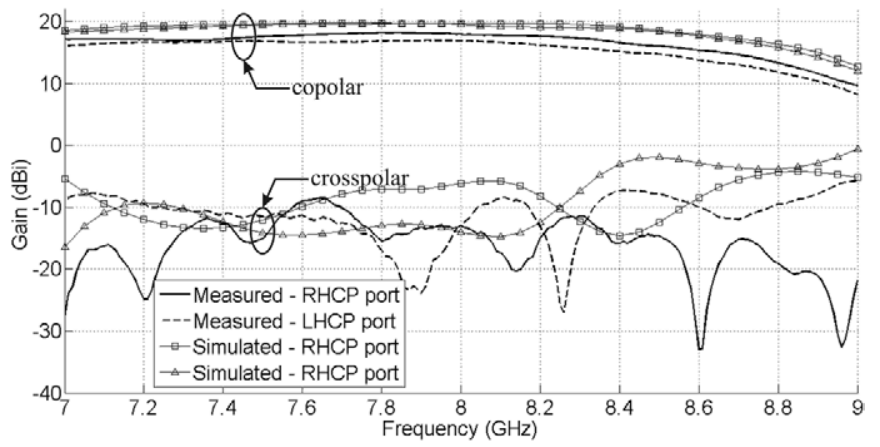

(a)

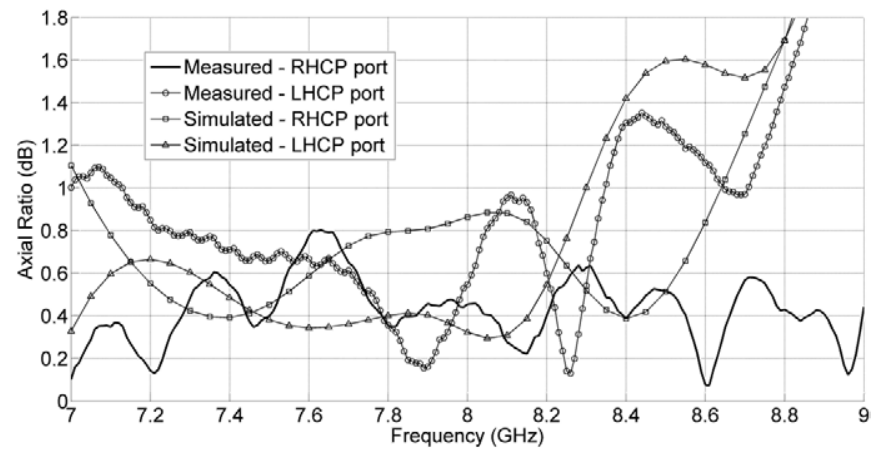

(b)

Fig. 8. Measured and simulated subarray results. (a) Gains of copolar and crosspolar components. (b) Axial ratios.

the coupling between microstrip lines will degrade the system behavior.

Because the LHCP network has a longer path than the RHCP one, higher losses, stronger couplings and worse input impedance matching will be expected in the former. Fig. 8 shows a comparison of the measured and simulated gains and axial ratios. A maximum gain of $18.2 \mathrm{dBi}$ for RHCP and approximately $17 \mathrm{dBi}$ for LHCP was measured for the copolar component in the central band. The minimum measured copolar gain is approximately $16.6 \mathrm{dBi}$ for RHCP and $15.2 \mathrm{dBi}$ for LHCP in the upper extreme band. LHCP displays between 1.2 and $1.4 \mathrm{~dB}$ of extra losses compared with RHCP. For RHCP, the losses compared to simulations are $1.5 \mathrm{~dB}$ in the center frequency and approximately $2.5 \mathrm{~dB}$ in the upper frequency. The copolar-crosspolar ratio is greater than $22.5 \mathrm{~dB}$ at all frequencies. The axial ratios of both polarizations remained below $1.4 \mathrm{~dB}$ over the entire bandwidth and agreed quite well with simulations.

The differences between simulations and measurements are more severe for LHCP due to higher losses and couplings in its feeding network. Because of these differences and unmodeled SMP connector losses, LHCP displayed worse behavior than RHCP.

To complete the set of subarray measurements, Fig. 9 shows the measured radiation patterns at $7.9 \mathrm{GHz}$ for both polarizations and for three cuts on the $\phi$ axis (azimuth).

\section{Power Distribution Network}

A stripline was used to distribute the signal among the subarrays, and it was designed to achieve a low-loss network 


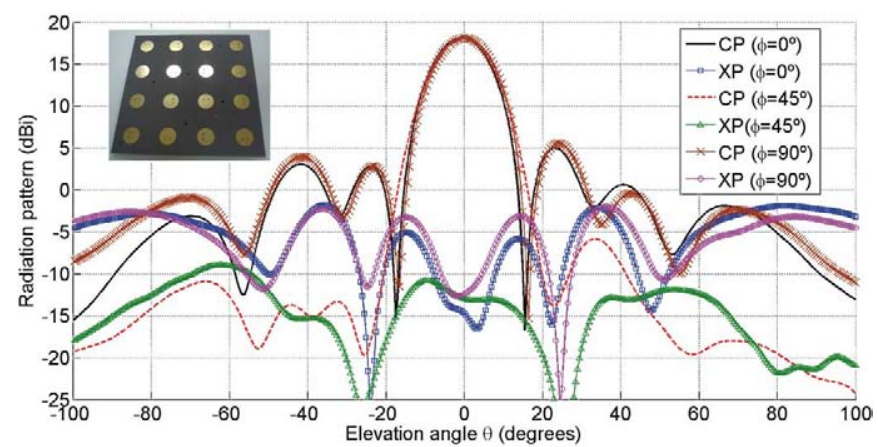

(a)

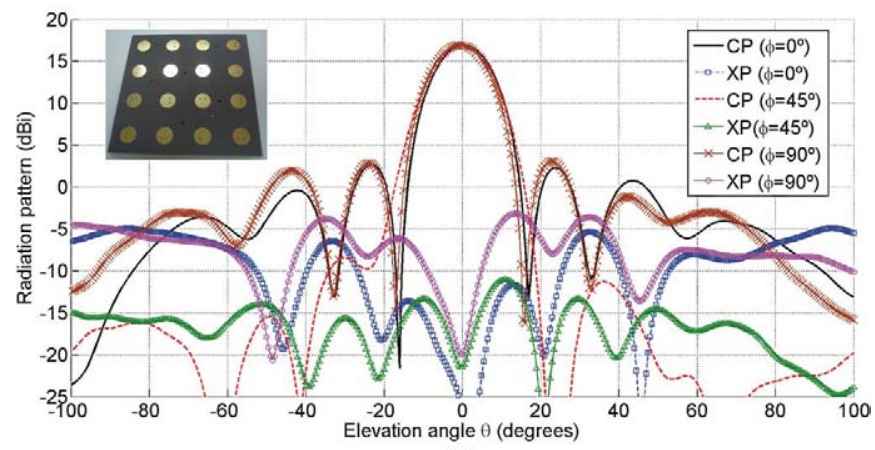

(b)

Fig. 9. Measured radiation patterns of the subarray at $7.9 \mathrm{GHz}$ for (a) RHCP and (b) LHCP. In the legend, CP refers to the copolar field component and XP to the crosspolar field component.

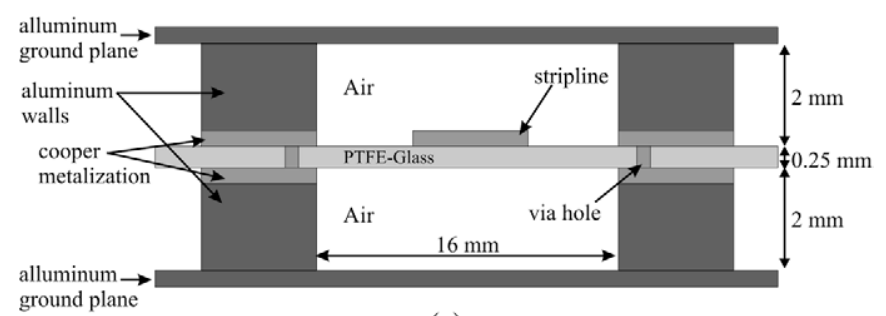

(a)

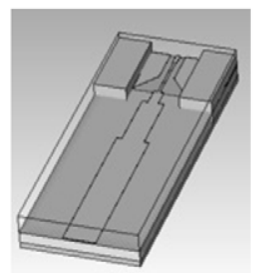

(b)

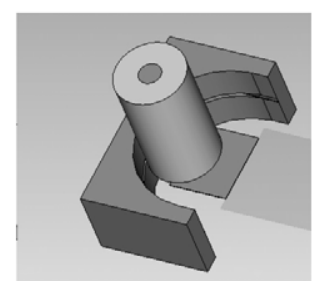

(c)
Fig. 10. Low-loss stripline. (a) Multilayer scheme side view. (b) Transition from stripline to SMP-type connector. (c) Transition from stripline to SMA-type connector.

and thus maximize the efficiency. The stripline is formed by a metalized line in a thin substrate suspended in air between two metallic plates, which act as ground planes. The air gap must be thick if low losses are required. A quasi-TEM mode is propagated through this transmission line utilizing the presence of two different dielectrics (PTFE-glass and air). However, to avoid the propagation of an undesired resonant TE mode, which can be excited by irregularities in the structure, the stripline is protected by surrounding it with metallic walls. This metallic structure also provides stiffness to the suspended stripline.

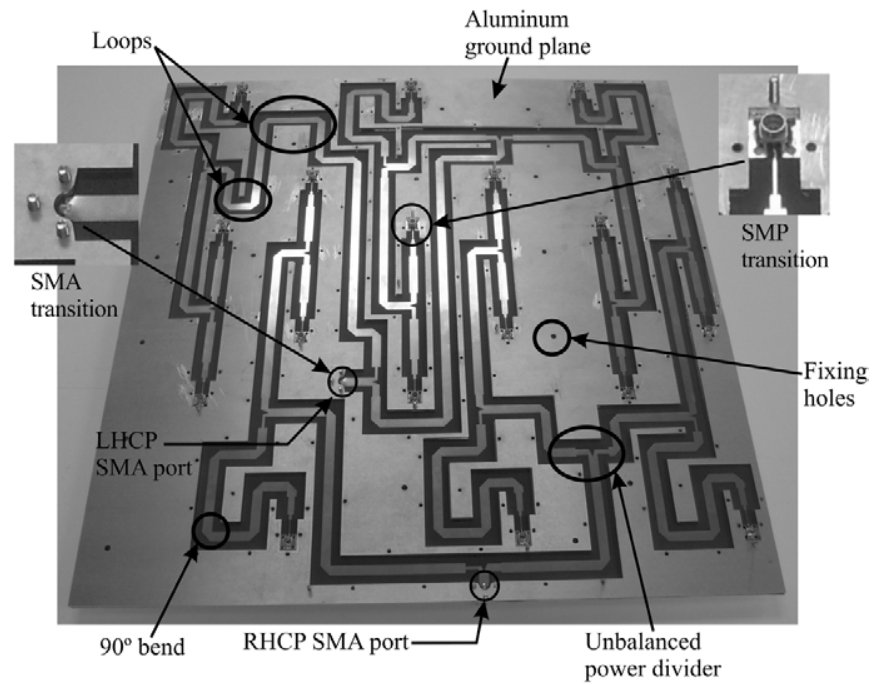

Fig. 11. Printed power distribution network board with aluminum walls.

The final structure is schematized in Fig. 10(a). The distance between the walls that shield the line has to be sufficiently small to prevent interference with the quasi-TEM mode and must also cut off the non-desired TE mode. A distance of 16 $\mathrm{mm}$ was set, and it was observed that the TE mode begins propagating above $9 \mathrm{GHz}$.

Transitions between the stripline and each subarray are performed through SMP-type connectors. There are two inputs/outputs in the antenna, one for each polarization, each of which has an SMA coaxial connector. These vertical transitions break the symmetry of the line, and to prevent the generation of a TE mode, it is necessary to protect them with a short-ended pseudo-waveguide that cuts off the TE mode. Fig. 10(b) and (c) show the protected transitions SMP and SMA, respectively. The losses are around 0.7 and $0.2 \mathrm{~dB}$ for the SMP and the SMA transitions, respectively. In addition, the SMP connectors must be mounted over a microstrip line; therefore, a transition between the stripline and microstrip line has to be designed to guarantee good impedance matching. The widths of the stripline and microstrip line are quite different, and the transition has to change the line width gradually as shown in Fig. 10(b).

Finally, the corporative network is depicted in Fig. 11, where the coaxial to stripline transitions are detailed. The network has two paths printed on the same layer, one for each polarization. The design has been performed, with a reference impedance of $50 \Omega$, branch by branch using loops and unbalanced power dividers to get the proper phase and amplitude in the subarray inputs, because the excitation levels of the subarrays are not equal, as was noted in Section II.

The measured relative power exciting each subarray is shown in Fig. 12 and Fig. 13 for RHCP and LHCP, respectively. The input power is used as the reference. Ideally, subarrays A, B and $\mathrm{C}$ should have relative power levels of approximately $-9,-10$ and $-11.2 \mathrm{~dB}$ (Fig. 12(a) and Fig. 13(a)), respectively, and must be excited in phase (Fig. 12(b) and Fig. 13(b)). This feeding configuration allows a lower SLL than a uniform excitation. 


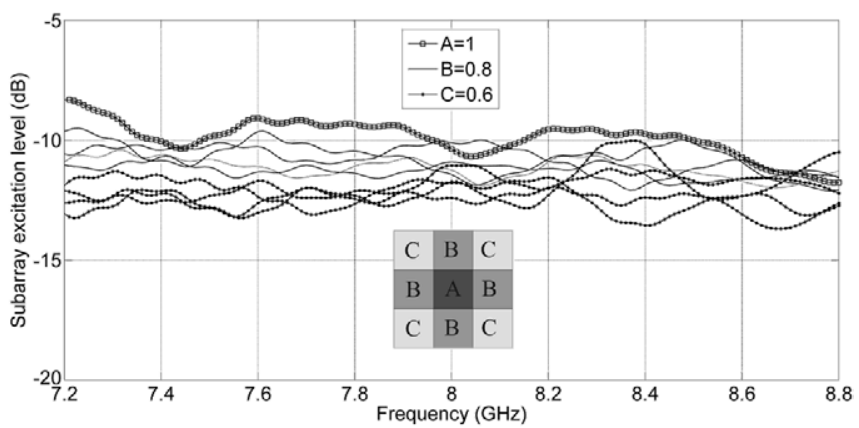

(a)

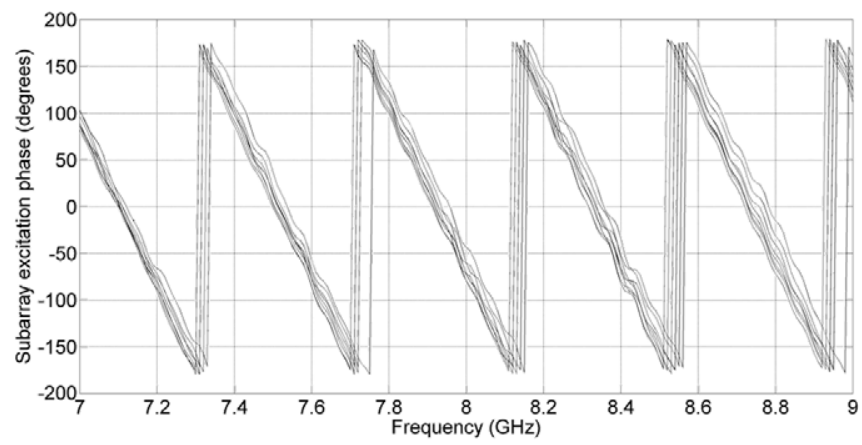

(b)

Fig. 12. Measured transmission parameters of RHCP to each subarray. (a) Amplitude. (b) Phase.

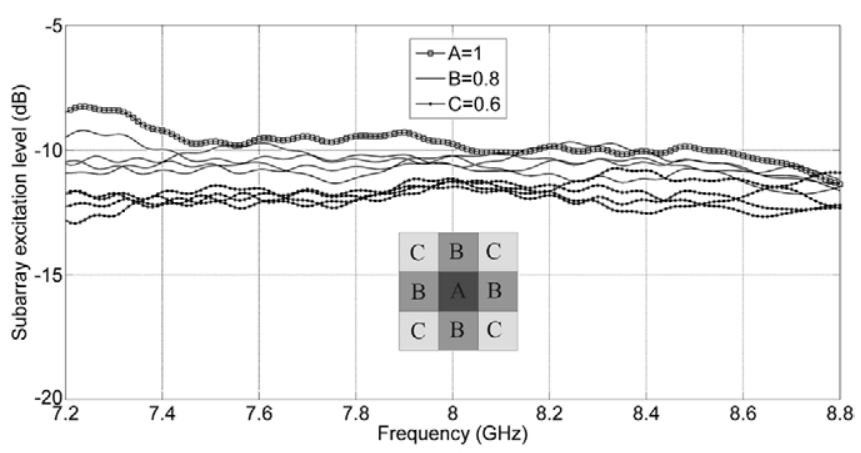

(a)

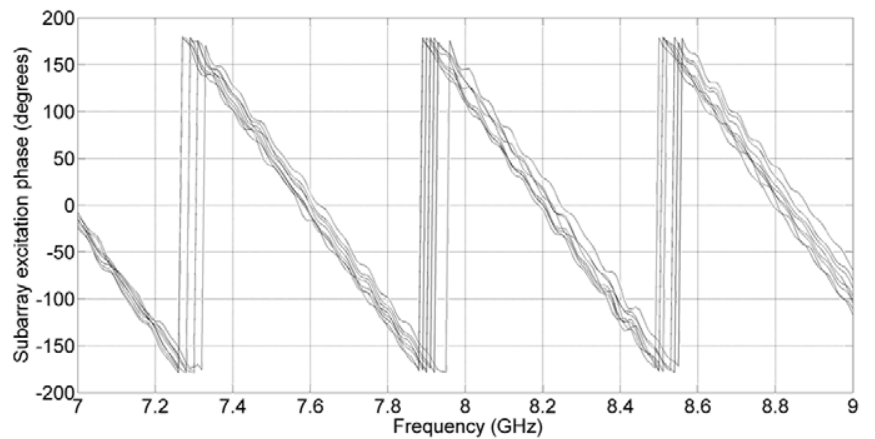

(b)

Fig. 13. Measured transmission parameters of LHCP to each subarray. (a) Amplitude. (b) Phase.

Fig. 14 exhibits the measured transmission losses of both polarizations, which are approximately $1.4 \mathrm{~dB}$ for LHCP and $1.8 \mathrm{~dB}$ for RHCP. Losses are higher in the latter because the paths are longer.

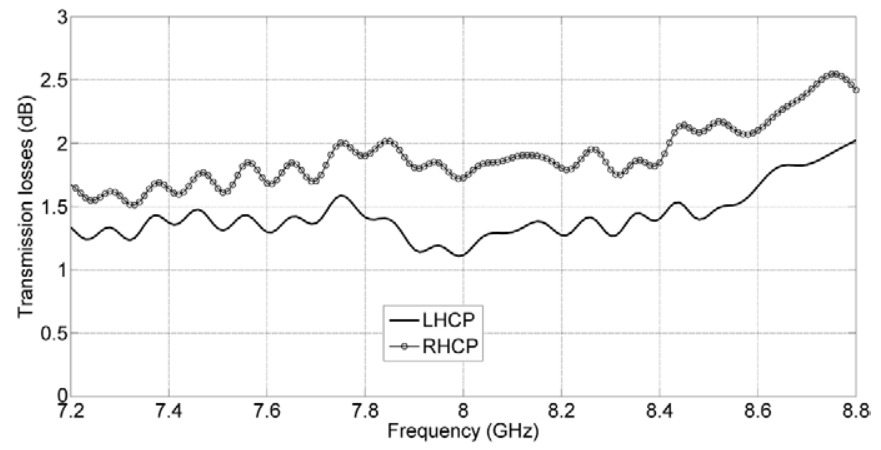

Fig. 14. Average measured transmission losses of both polarizations in the power distribution network from SMA global connectors to SMP subarray input ports.

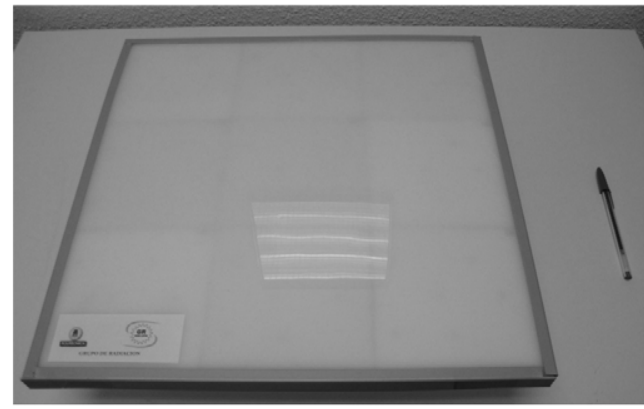

(a)

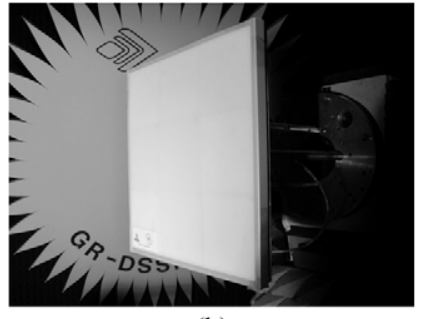

(b)

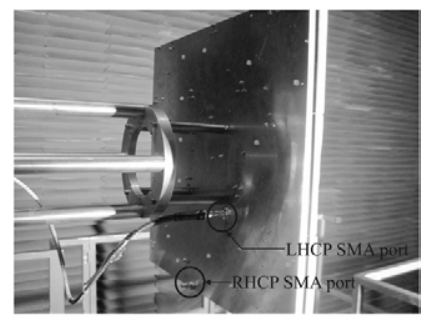

(c)
Fig. 15. (a) Antenna system with radome. (b) Antenna in anechoic chamber, frontal view. (c) Antenna in anechoic chamber, back view with SMA connectors.

\section{RESULTS AND DisCUSSION}

The complete antenna was measured successfully. Fig. 15 depicts the final antenna system with a PVC radome mounted in an anechoic chamber for measurement acquisition.

Fig. 16 shows the measured S-parameters of the two SMA ports. The input reflection coefficient remains under $-10 \mathrm{~dB}$ over the entire band, which is sufficient to ensure a radiation efficiency above $50 \%$. The observed peaks are mainly due to strong couplings in the subarray feeding network. The coupling between ports is under $-20 \mathrm{~dB}$ in the working band.

Fig. 17 shows the measured and simulated values of the realized gain and axial ratio. The maximum measured gain for the copolar component is $26.2 \mathrm{dBi}$ for RHCP and $25.8 \mathrm{dBi}$ for LHCP in the central band. The measured minimum copolar gains in the upper frequency band are 24.5 and $23.8 \mathrm{dBi}$ for RHCP and LHCP, respectively. LHCP has between 0.4 and $0.7 \mathrm{~dB}$ extra losses compared to RHCP because of its stronger couplings inside the microstrip feeding network and higher 


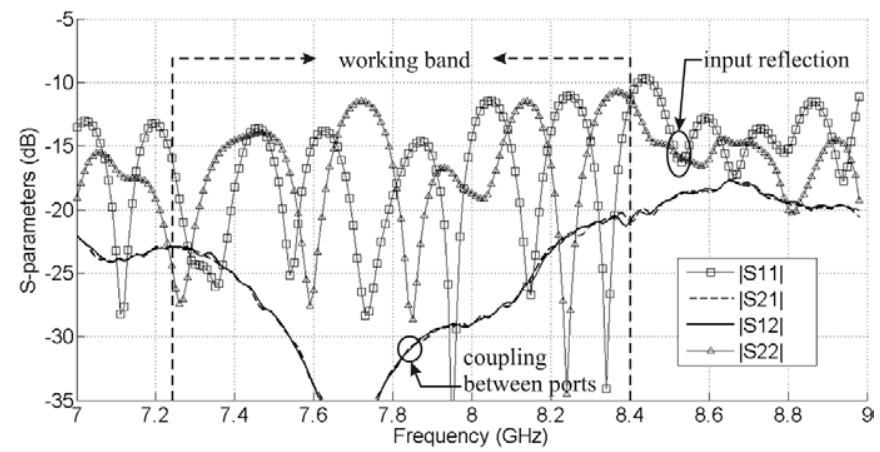

Fig. 16. Measured S-parameter amplitudes for the antenna, where port 1 is the SMA LHCP input/output and port 2 is the SMA RHCP input/output.

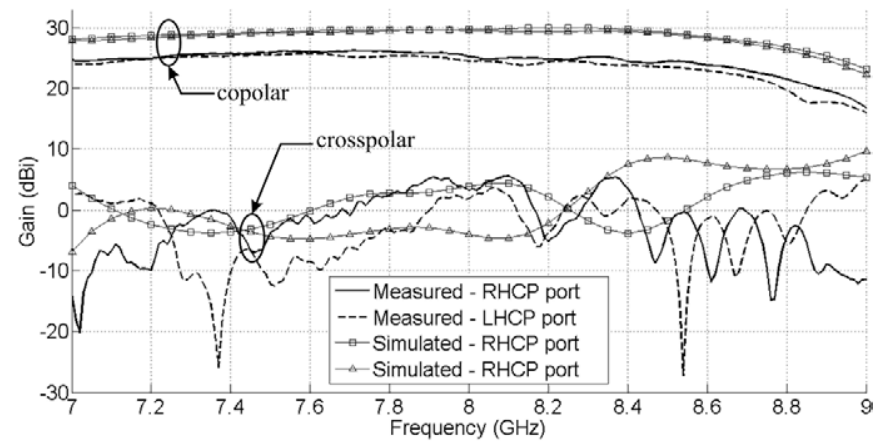

(a)

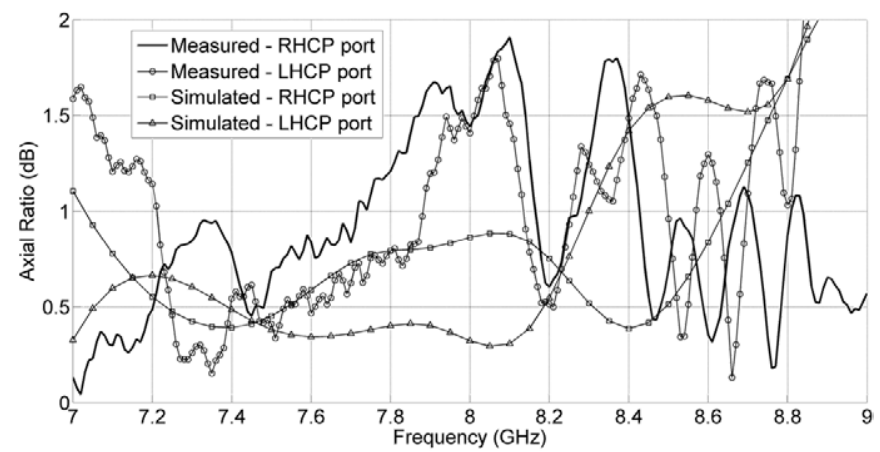

(b)

Fig. 17. Measured and simulated antenna results. (a) Gains of the copolar and crosspolar components. (b) Axial ratios.

transmission losses due to its longer paths, as was already mentioned in Sections III and IV. The measured and simulated gains for RHCP, exhibit $3.5 \mathrm{~dB}$ of difference at the center frequency and approximately $5.2 \mathrm{~dB}$ at the upper frequency. These losses are primarily due to the transitions between the SMA/SMP connectors and the printed lines, which were not considered in the simulations. Furthermore, the simulations were performed without the global stripline network, and the losses in Fig. 14 were therefore not included and must be subtracted.

The measured gain guarantees a radiation efficiency of 50\% for this antenna system, measured at $8.25 \mathrm{GHz}$ for LHCP. The maximum measured radiation efficiency is $60 \%$ for the RHCP port at $7.75 \mathrm{GHz}$. Finally, the copolar-crosspolar ratio is above $20 \mathrm{~dB}$ over the entire band, and the axial ratio remains below $1.9 \mathrm{~dB}$ for both polarizations.

Figs. 18, 19 and 20 show the measured radiation patterns at 7.25, 7.9 and $8.4 \mathrm{GHz}$, respectively, for the two polarizations
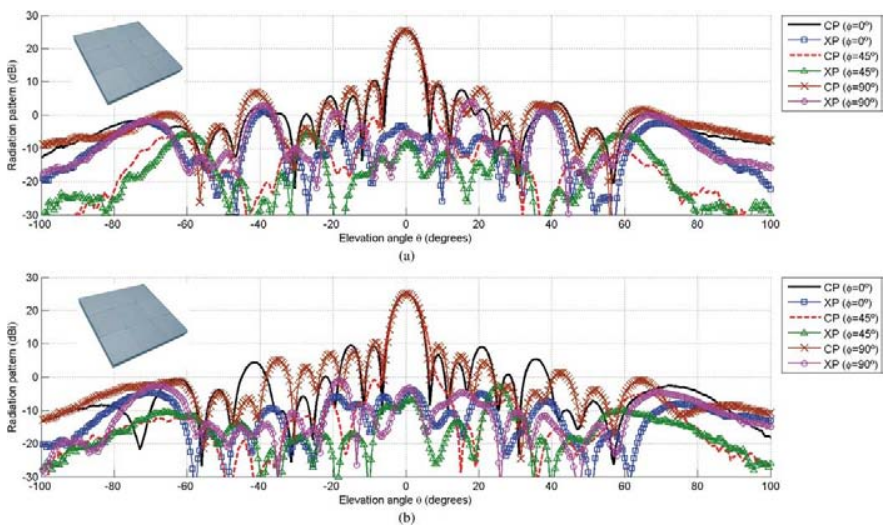

Fig. 18. Measured radiation pattern of the antenna at $7.25 \mathrm{GHz}$ for (a) RHCP and (b) LHCP. In the legend, CP refers to the copolar field component and XP to the crosspolar field component.

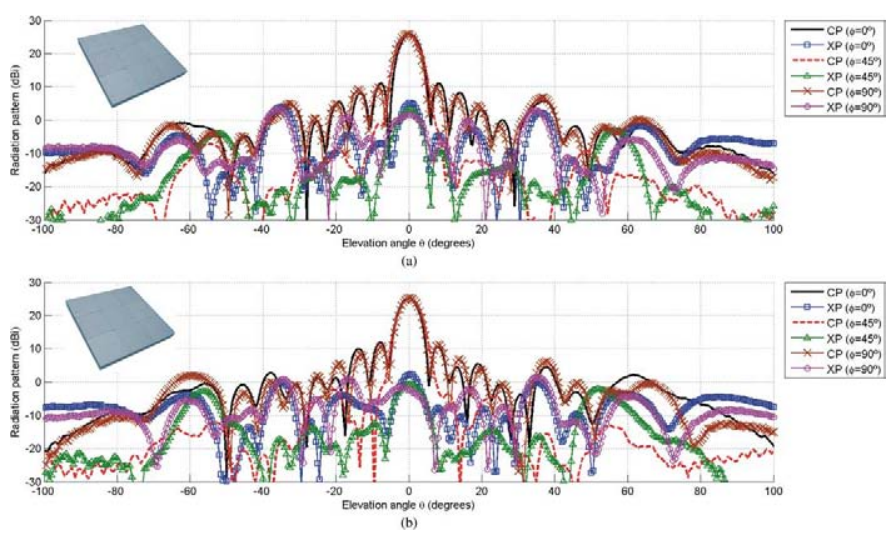

Fig. 19. Measured radiation pattern of the antenna at $7.9 \mathrm{GHz}$ for (a) RHCP and (b) LHCP. In the legend, CP refers to the copolar field component and XP to the crosspolar field component.
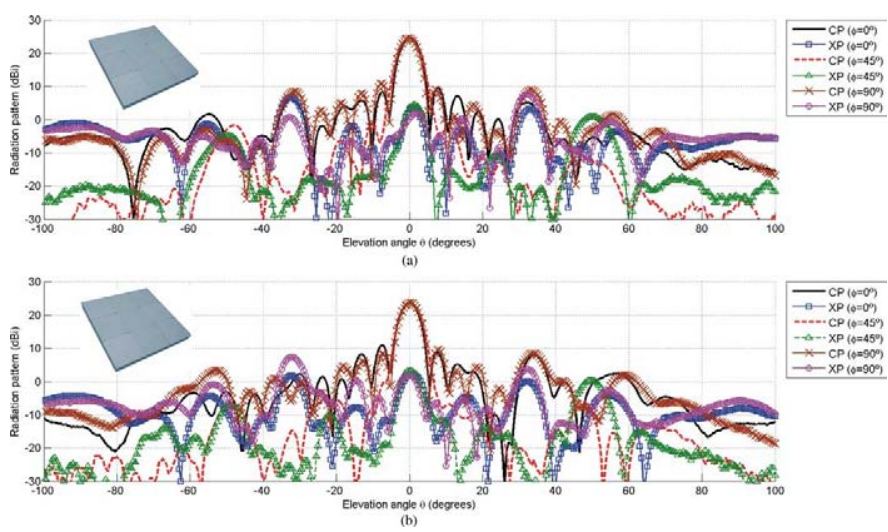

Fig. 20. Measured radiation pattern of the antenna at $8.4 \mathrm{GHz}$ for (a) RHCP and (b) LHCP. In the legend, CP refers to the copolar field component and XP to the crosspolar field component.

and for three cuts on the $\phi$-axis. SLL values are less than -15 and $-13 \mathrm{~dB}$ for RHCP and LHCP, respectively, at all frequencies.

The good performance of this antenna system shows that the infinite array approximation for the radiating element design is sufficiently accurate for this array size. This approach is very important to prevent couplings among elements, and it makes possible to achieve active impedance matching when a 
high dielectric thickness is unavoidable to fulfill the bandwidth requirements.

\section{CONCLUSION}

In this paper, the complete design and characterization of a printed antenna for X-band satellite communications (7.25-8.4 GHz) were presented. This antenna can work as a portable man-pack for personal satellite communication services, and its main advantages are its low profile, modularity, high efficiency and wide frequency band.

The array is divided into 9 equal subarrays of $4 \times 4$ doublestacked microstrip patches. A BLC allows it to have dual circular polarization, which was miniaturized to save space in the subarray feeding network and reduce the couplings between the lines. The subarrays have microstrip feeding structures, and each subarray is fed with a low-loss protected stripline. Transitions between subarrays and the global corporative network are performed through SMP-type coaxial connectors. There are also two SMA ports, one for each polarization, which are the global input/output. These transitions have to be treated carefully to avoid the excitation of undesired modes. The sequential rotation technique is applied between the two by two elements to form the unit cell of the array and provide more pure circular polarization. To minimize the SLL, the subarrays have excitation levels that decrease from the center to edges.

Measurements show that the gain is between 23.8 and $26.2 \mathrm{dBi}$, the radiation efficiency is between $50 \%$ and $60 \%$, the copolar-crosspolar ratio is above $20 \mathrm{~dB}$, the axial ratio is below $1.9 \mathrm{~dB}$ and the SLL is less than $-13 \mathrm{~dB}$. The measured and simulated results are in very strong agreement given that the effects of the global distribution network and transitions between the coaxial connectors and lines are not considered in the simulations. Therefore, because the measured losses of such transitions are around 1 or $2 \mathrm{~dB}$ and the stripline network losses are between 1.4 and $1.8 \mathrm{~dB}$, differences of approximately 3 to $6 \mathrm{~dB}$ between simulations and measurements of the complete antenna were expected.

\section{ACKNOWLEDGMENT}

The NY substrate used in the prototypes was kindly provided by NELTEC S.A.

\section{REFERENCES}

[1] B. G. Evans, Satellite Communication Systems, 3rd ed. London, U.K.: The Institution of Engineering and Technology, 2008.

[2] M. Sierra-Castañer, M. Vera-Isasa, M. Sierra-Pérez, and J. L. Fernández-Jambrina, "Double-beam parallel plate slot antenna," IEEE Trans. Antennas Propag., vol. 53, no. 3, pp. 977-984, Mar. 2005.

[3] A. Stark, A. Dreher, H. Fischer, A. Geise, R. Gieron, M. Heckler, S Holzwarth, C. Hunscher, A. F. Jacob, K. Kuhlmann, O. Litschke, D. Lohmann, W. Simon, F. Wotzel, and D. Zahn, "SANTANA: Advanced electronically steerable antennas at Ka-band," in Proc. 3rd EuCAP, Berlin, Germany, 2009, pp. 471-478.

[4] D. A. Bajgot, "Development of a low-cost, man-portable, phased array X-band radar," in Proc. 2010 IEEE Int. Symp. ARRAY, Waltham, MA, 2010, pp. 183-188.

[5] W. H. Theunissen et al., "Development of an X-band phased array antenna," in Proc. 2011 IEEE Int. Symp. ARRAY, Waltham, MA, 2010, pp. 211-218.
[6] S. Vaccaro, F. Tiezzi, M. F. Rúa, and C. D. G. De Oro, "Ku-band lowprofile Rx-only and Tx-Rx antennas for mobile satellite communications," in Proc. 2010 IEEE Int. Symp. ARRAY, Waltham, MA, Oct. 2010, pp. 536-542.

[7] R. Mailloux, J. McIlvenna, and N. Kernweis, "Microstrip array technology," IEEE Trans. Antennas Propag., vol. 29, no. 1, pp. 25-37, Jan. 1981.

[8] J. R. James and P. S. Hall, Handbook of Microstrip Antennas. London, U.K.: Peter Peregrinus, 1989.

[9] W. Wu, J. Yin, and N. Yuan, "Design of an efficient X-band waveguide-fed microstrip patch array," IEEE Trans. Antennas Propag., vol. 55, no. 7, pp. 1933-1939, Jul. 2007.

[10] R. Garg, P. Bhartia, I. Bahl, and A. Ittipiboon, "Circularly Polarized Microstrip Antennas and Techniques," in Microstrip Antenna Design Handbook. Norwood, MA: Artech House, 2001, ch. 2, pp. 493-532.

[11] L. Infante, A. De Luca, and M. Teglia, "Low-profile ultra-wide band antenna array element suitable for wide scan angle and modular subarray architecture," in Proc. 2010 IEEE Int. Symp. ARRAY, Waltham, MA, 2010, pp. 157-163.

[12] Y. Kuwahara, Y. Matsuzawa, H. Kitahara, and M. Haneishi, "Phased array antenna with a multilayer substrate," IEE Proc. Microwaves, Antennas Propag., vol. 141, no. 4, pp. 295-298, Aug. 1994.

[13] H. F. Pues and A. R. Van de Capelle, "An impedance-matching technique for increasing the bandwidth of microstrip antennas," IEEE Trans. Antennas Propag., vol. 37, no. 11, pp. 1345-1354, Nov. 1989.

[14] N. C. Karmakar, "Investigations into a cavity-backed circular-patch antenna," IEEE Trans. Antennas Propag., vol. 50, no. 12, pp. 1706-1715, Dec. 2002.

[15] D. Sievenpiper, L. Zhang, R. F. J. Broas, N. G. Alexopolous, and E. Yablonovitch, "High-impedance electromagnetic surfaces with a forbidden frequency band," IEEE Trans. Microw. Theory Tech., vol. 47, no. 11, pp. 2059-2074, Nov. 1999.

[16] G. L. Matthaei, L. Young, and E. M. T. Jones, “TEM-mode, coupledtransmission-line directional couplers and branch-line direcitional couplers," in Microwave Filters, Impedance-Matching Networks, and Coupling Structures. Norwood, MA: Artech House, 1980, ch. 13, pp. 775-842.

[17] W.-L. Chen and G.-M. Wang, "Exact design of novel miniaturised fractal-shaped branch-line couplers using phase-equalising method," IET Microwaves, Antennas Propag., vol. 2, no. 8, pp. 773-780, Dec. 2008.

[18] Z. Liu and R. M. I. Weikle, "A compact quadrature coupler based on coupled artificial transmission lines," IEEE Microw. Wireless Compon. Lett., vol. 15, no. 12, pp. 889-891, Dec. 2005.

[19] C. Tang and M. Chen, "Synthesizing microstrip branch-line couplers with predetermined compact size and bandwidth," IEEE Trans. Microw. Theory Tech., vol. 55, no. 9, pp. 1926-1934, Sep. 2007.

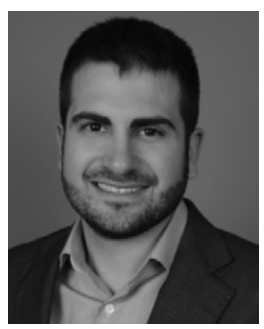

Andrés García-Aguilar was born in Madrid, Spain, in 1984. He received the B.Sc. and M.Sc. degrees in telecommunications engineering from Technical University of Madrid (UPM), Spain, in 2008 and 2009, respectively, where he is currently working toward the Ph.D. degree in the Radiation Group, Signals, Systems and Radiocommunications Department as a recipient of a Spanish Ministry of Science and Innovation scholarship (2009-2013).

In 2011, he was with the Faculty of Electrical Engineering and Computing, University of Zagreb, Croatia, as invited Ph.D student. His research interests are planar and conformal antenna design, numerical methods and metamaterials applied to antenna and microwave device analysis and synthesis.

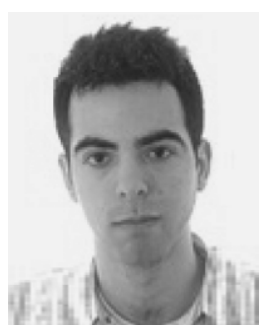

José-Manuel Inclán-Alonso was born in Madrid, Spain, in 1986. He received the B.Sc degree in telecommunications engineering from Technical University of Madrid (UPM), Spain, in 2010, where he is currently working toward the Ph.D degree in the Radiation Group in the Signals, Systems and Radiocommunications Department.

His research interests include array design at $\mathrm{X}$ and $\mathrm{Ka}$ Band for satellite communications and electromagnetic numerical methods in time domain. 


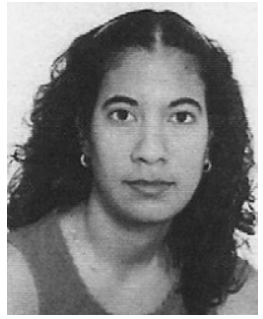

Lucía Vigil-Herrero was born in Madrid, Spain, in 1981. She received the B.Sc. degree in telecommunications engineering from Technical University of Madrid, Spain, in 2011.

Her main research activity is in antenna synthesis and design for satellite communications at microwave frequencies.

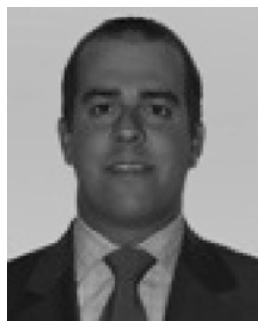

José-Manuel Fernández-González was born in Lausanne, Switzerland, in 1977. He received the Diplôme d'Ingénieur en Électricité from the École Polytechnique Fédérale de Lausanne (EPFL), Switzerland, in 2003. From October 2002 to March 2003, he completed the M.S. thesis as an Erasmus program student in the Radiation Group, Signals, Systems and Radiocommunications Department at Technical University of Madrid, where he received the Ph.D degree in the Radiation Group, Signal, Systems and Radiocommunications Department.

From January to May 2006, he has spent three months abroad as a guest Ph.D student at "Centre de Recherches Poly-Grames" of l'École Polytechnique de Montréal, QC, Canada. From September to December 2007, he was with the Antenna Group, Signals and Systems Department at Chalmers University of Technology, Göteborg, Sweden as invited Ph.D student. Since 2009, he is a postdoctoral researcher in the Radiation Group, Signals, Systems and Radiocommunications Department at Technical University of Madrid. His current research interest is in the field of metamaterial structures with emphasis on planar antenna applications. He has been active in the area of antenna synthesis and design, and participates actively in national and international investigation projects for companies.

Dr. Fernández has been the recipient of a Spanish Ministry of Education and Science scholarship (2006-2010).

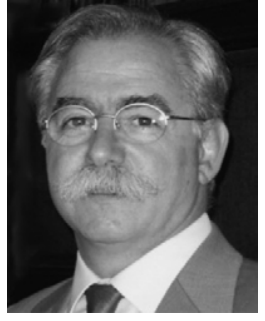

Manuel Sierra-Pérez (M'90-SM’98) was born in Zaragoza, Spain, in 1952. He received the M.S degree (Telecommunication engineer) and the $\mathrm{PhD}$ degree from the Technical University of Madrid (UPM), Spain, in 1975 and 1980, respectively.

He became Full Professor in the Signal, Systems and Radiocommunications Department, UPM, on January 1990. He has led a large number of research projects with public organizations and companies. $\mathrm{He}$ is the author of several books and book chapters and review papers about the research projects. He has been working as invited Professor at the NRAO, VA, in 1980 and at the University of Colorado in 1995. His research experience and main interest is in the area of antennas and radiofrequency circuits associated to the antennas. Particularly, the main research projects in the last years have been centered in the design of antenna arrays, either active or passive, and associated circuits, from the passive networks to the full radiofrequency receivers and transmitters.

Prof. Sierra-Pérez has been the chairman of the European Conference on Antennas and Propagation (EuCAP-2010) celebrated in Barcelona (Spain). He has been president of the IEEE Spain Section and president and founder of the IEEE Spain AP and MTT chapter. He is member of the EuCAP steering committee, member of EuRAAP on behalf of Spain and Portugal, member of the IEEE Spain Section Committee, member of Spain URSI committee and national URSI conference organization committee. He is the Spain delegate in the COST IC-0603 ASSISTS project. 